\title{
The Role of Adaptive Mission Planning and Control in Persistent Autonomous Underwater Vehicles Presence
}

\author{
Mario Paulo Brito \\ Marine Autonomous Robotic Systems \\ National Oceanography Centre (NOC) \\ Southampton, United Kingdom \\ mario.brito@noc.ac.uk
}

Ron Lewis

Marine Environmental Research Lab for Intelligent Vehicles Memorial University of Newfoundland

St. John's, Canada

ron@mun.ca

Gwyn Griffiths

Marine Autonomous Robotic Systems

National Oceanography Centre (NOC)

Southampton, United Kingdom

gxg@noc.ac.uk

\begin{abstract}
The Autonomous Underwater Vehicle (AUV) community has for many years recognized the potential benefits made by adapting mission planning on-the-fly. Over the years there has been some degree of success in applying adaptive mission planning to very specific problems. Examples of applications include capabilities for a vehicle to search for, and then modify its trajectory to follow, a feature such as a plume or a thermocline, or to modify its trajectory to avoid an obstacle, or to find and follow a feature such as a pipeline. Despite an evident increase in the number of applications, the use of adaptive mission planning is still in its infancy. There is no doubt that adaptive mission planning will play a pivotal role in future AUV persistent presence. So what is delaying this technology from making the leap towards wider industry acceptance? This paper reviews the literature in adaptive mission planning and uses a failure analysis technique to identify key obstacles for the integration of this technique in wider AUV applications. We use our failure analysis to help devise recommendations for mitigating these obstacles. The complexity of the mathematical approaches used by adaptive techniques is one key obstacle. Perhaps of more importance is that the AUV community is increasingly requiring quantitative assessment of risk associated with the use of AUVs. We propose that probability is the appropriate measure for quantifying the risk of adaptive systems and their uncertainty. The work here presented is a collective endeavor of the Engineering Committee on Oceanic Resources Specialist Panel on Underwater Vehicles.
\end{abstract}

Index Terms-autonomous underwater vehicles, adaptive mission planning, risk, precision, positioning.

\author{
Neil Bose \\ Australian Marine College (AMS) \\ University of Tasmania, \\ Launceston, Australia \\ nbose@amc.edu.au
}

Polly Alexander

Tasmanian ICT Centre, Commonwealth Scientific and Industrial Research Organisation (CSIRO)

Hobart, Australia

polly.alexander@csiro.au

James Ferguson

International Submarine Engineering

Port Coquitam, Canada

james.ferguson@ise.bc.ca

\section{INTRODUCTION}

The research presented in this paper is a result of a collective endeavor of the Engineering Committee on Oceanic Resources (ECOR) Specialist Panel on Underwater Vehicles (SPUV) to understand the potential role of adaptive mission planning in persistent autonomous underwater presence.

By now a number of nations share the vision that autonomous underwater vehicles (AUVs) provide an increasingly capable solution for ocean sampling and surveillance systems [1][2]. As a result many commercial manufacturers are now able to supply the growing market. Curtin et al. [3], argue that a network of autonomous underwater vehicles is a low cost solution able to meet the following objectives: global deployment; sustained presence; three dimensional adaptive performance and real time control and robust performance.

Indeed major advances in energy consumption and communications have led to the development of a new generation of AUVs that permit global deployment, sustained presence and real time control and robust performance. However the use of adaptive mission planning is not as widely adopted as originally envisaged.

Researchers in the field of mission control of autonomous underwater vehicles have for many years recognized the potential gains to be made by adapting the mission plan of the vehicle on-the-fly. Examples of mission plan changes might be for a vehicle to search for and then modify its trajectory to follow a characteristic, such as an outfall plume or a 
thermocline [4]; it might be for a vehicle to have to modify its path to avoid an obstacle, or to search for, find and follow a feature, such as a buried pipeline; or it might be for an action to be taken by a vehicle when a particular value is registered on a sensor, such as the collection of a water sample if the concentration of a particular chemical reaches a certain value, or for fault tolerance [5][6].

However, the use of adaptive mission planning systems is still in its infancy. As AUVs evolve to address more ambitious mission requirements the key question that needs to be addressed is, can we rely on adaptive mission technology for AUV mission planning on the fly?

There are many potential causes for the failure to adopt a given technology. The objective of this paper is to identify the main reasons that may be impeding greater use of adaptive mission systems so that we can identify potential mitigation actions that can be used by AUV stakeholders, developers, industry, or marine science users. We have conducted a formal investigation into the reasons causing failure to adopt mission adaptive systems. All hypotheses are captured in a structured event tree. In a similar style to a formal accident investigation, a panel of experts was asked to assess the evidence and estimate the most likely causes for failure to adopt mission planning technology. Ten experts took part in this study; experts comprised adaptive systems scientists, AUV engineers, managers and technologists.

This paper is organized as follows. Section II presents a review of adaptive mission planning systems, focusing on those used in oceanography. Section III presents the failure analysis process adopted to support this research. Section IV presents an analysis of the experts' assessments. Section V presents the discussions and recommendations. Finally section VI presents our conclusions.

\section{REVIEW OF MISSION ADAPTIVE SYSTEMS}

Traditional robot decision making is based on three distinct processes: Sense, Plan, and Act (SPA). With the move towards enhanced decision making, perception, and intelligent capabilities, there has been an effort to extend and redesign the decision making architecture to facilitate development of these new robotic systems. At this stage, it is important to define what we mean by intelligence. There are many definitions of intelligence; the most widely accepted definition, and adopted in this publication, is that presented by Mystel et al. in [7]: 'Intelligence is the ability of a system to act appropriately in an uncertain environment, where an appropriate action is that which increases the probability of success, and success is the achievement of behavioural sub-goals that support the system's ultimate goal.'

Several authors have proposed different architectures for mission adaptive systems. Albus et al. [8] propose a model of an "intelligent machine system" that lays out a system with situational assessment feeding information on world models and value judgments that lead to behaviour generation. These are key concepts that are recurrent in many of the architectures and decision systems that have been proposed since. John Boyd [9], a military strategist, proposed a four level decision cycle [10]: Observe; Orient; Decide; Act. Referred to as the OODA cycle this has been applied across many applications in business, the military, robotics, and others. Patron et al. [11][12][13] apply this architecture to an autonomous mission plan recovery system designed to repair plans and create new plans depending on the situation. In this, application situational awareness encapsulates both the Observe and Orient components of Boyd's cycle, while a planner using analytical tools makes the 'Decisions' and a low level interface facilitates the 'Actions'. In a paper by Evans et al. [13] this four level decision making cycle was combined with the traditional SPA cycle and is re-implemented as: Reactive layer; Scenario layer; Sensor layer; Mode layer.

In particular, these authors were looking at collision avoidance for autonomous robots and escape as a subsumption ${ }^{1}$ approach where the reactive layer still provides a fast emergency response [14]. Dabe et al. [15] proposed a four level cycle consisting of: Perception; Situation Evaluation; Decision; Action.

The difference between these different control loops is the decision making cycle. There is no argument about sensing and acting as critical stages in the process, the question remains, how does a robot decide what action to take given particular sensor data and user inputs? For this there are two distinct schools of thought. One framework follows a probabilistic approach, where feature tracking is achieved by having the machine learn hidden Markov model chains (HMM) from past AUV runs. A large number of scenarios are needed in order to train the HMM to make good estimates of the current environment state. When in a real mission the vehicle will act upon the most likely scenario, as estimated by the HMM. A second framework looks at the problem from an optimization perspective, where for a given set of observations the system reaches a decision that maximizes the utility function. Given that there are a number of possible behaviours this is often a multiple objective optimization problem - different approaches are proposed to solve this problem.

There are several existing frameworks for adaptive mission systems that are currently being used and developed for AUVs and spacecrafts. Some well known frameworks are: MOOSIvP [16], TREX [17], ORCA [18], and ROS[19]. The predominantly implemented frameworks are T-REX and the MOOS-IvP. They will be reviewed here. A review of other frameworks is presented in [20].

T-REX (or the Teleo-Reactive EXecutive) was developed based on the temporal constraint-based planner EUROPA developed at NASA [21][22]. T-REX is a modular control

1 Is a system coordination method where the architecture is built incrementally by adding layers in different phases. Each layer is composed of one or more Augmented Finite State Machines (AFSM), and depending on sensory information the layer can be active or not. When a layer is active, its output suppresses all outputs from the layers below taking the control of the vehicle. The layer can remain active for a period after the activation conditions finishes. It reduces the robot's control architecture into a set of task-achievement behaviours or competences. Individual layers work on individual goals concurrently and asynchronously. Layers are organised hierarchically allowing higher layers to inhibit inputs or suppress outputs of lower layers. 
system with one 'Teleo-reactive agent' or coordinator that is made up of many semi-independent Sense Plan Act (SPA) controllers, known as Teleo-Reactors. Each of these TeleoReactors, or reactors, has a different functional and temporal scope. Functional scope for a reactor is a particular set of goals it understands, can plan action to achieve, and observe the state of actions to monitor goal success. Temporal scope here is the amount of time ahead for which a reactor makes a plan or deliberates, and latency is the amount of time it is allowed to take to make the plan. Each reactor has a 'timeline' for each of the actions that are within its functional scope. These timelines are used to store the previous state of the action and plan the action state into the future. The reactors also have the facility to pass and receive goals for action upon, and observe the state recorded in the timeline of other reactors to access the state of their requested goals. There are four timelines:

1. A Transect Timeline that takes locations and driving patterns.

2. A Path Timeline that takes latitude, longitude and depth.

3. A Command Timeline that understands commands for the vehicle control, (e.g. speed, rudder angle).

4. A Position Timeline that reports the vehicle position.

Each reactor has its own internal and external observing timelines that allow goals to be sent from one reactor to another.

In a given deployment the "Mission Manager" reactor would receive goals from the user, such as science features to watch for, rendezvous points and mission timeouts. Its temporal scope would be the entire mission, and latency would be several minutes. This Mission reactor makes an initial plan for the entire mission where its actions are goals that are passed to the "Science Manager" and "Navigation Manager" reactors. An example goal for the Science Manager might be to identify thermoclines (dramatic vertical changes in water temperature), and for the "Navigation Manager" an initial waypoint request (go to latitude, longitude, depth). The Science Manager may then produce a plan to follow a path at a certain depth to check for a thermocline. It puts this plan as a request on "Transect" timeline that the "Mission Manager" has control over. These two middle level reactors might make plans for a minute ahead at a time, and would only need a second of latency to make these plans. Example actions that the Science reactor might produce, in response to a state read from the Vehicle Command Manager are requests for different paths between waypoints or diving patterns from the Navigation Manager or deployment of a special sensor from the Vehicle Command Manager.

In [23] Rajan et al. present the results of two experiments using T-REX. Both were volume surveys. The first was an Intermediate Nepheloid Layers (INL) survey, and the purpose of the second survey was to track ocean fronts delineated by thermal gradients. The T-REX agent ran on a $367 \mathrm{MHz}$ EPXGX500 AMD Geode stack using Red Hat Linux within a $10 \mathrm{~Hz}$ control loop, the functional layer ran on a separate processor using real-time QNX on the Dorado AUV. The scientific payload for this AUV was: two CTDs, a HobiLabs HS2 HydroScat optical backscatter sensor and a Satlantic/MBARI
ISUS Nitrate sensor. The experiments were carried out in depths up to $100 \mathrm{~m}$ [23].

The INL survey was carried out in November 2008 over the Monterey Canyon. The vehicle performed a 6 hour and a 40 minute mission. The vehicle was fitted with ten water samplers, two water samplers were operated simultaneously enabling collection of 2 litre samples every two minutes. Results of this deployment show that the vehicle had changed its course in order to maximize the chances of capturing INL. The second deployment took place close to Monterey Bay. This involved a human-in-the-loop mixed initiative, where the scientist, in a location offshore, would indicate areas of interest. The vehicle navigated to these areas to locate the centroid of the front. Communication with the vehicle was via the Iridium satellite network.

MOOS-IvP is a behaviour based mission management system within a software framework and toolset designed to resolve optimal action for an autonomous system with competing subsystem goals. The Mission Oriented Operating Suite (MOOS) is a software framework to facilitate modular development of software for autonomous systems. It is based around the construction of modular software processes that communicate using a publish/subscribe communications protocol, where modules can publish information from themselves and subscribe to publications made by other software modules via a central database. By creating publicly available middleware for modular software design, MOOS facilitates a community of shared work, rapid shared development of tools and infrastructure, and a common architecture for collaboration.

MOOS-IvP is a software bundle that contains a snapshot of a MOOS open source release version with the IvP Helm, a MOOS process supported by the Massachusetts Institute of Technology (MIT). The IvP Helm uses a behavioural-based architecture to organize its decision making. Each behaviour produces an IvP objective function that is used by the solver to make its decisions. It resolves competition between different behaviours by performing multiple objective optimization on their collective output using a mathematical programming model called interval programming (IvP).

A MOOS-IvP mission consists of a set of user configurable behaviours, a state space model for activation and transition of behaviours. The modular nature of MOOS and the IvP Helm is based around the objective of code re-use in terms of code interoperability between vehicles and deployments and the benefits of code security with core code being used for substantial amounts of time in the field. Five benefits of code reuse are identified: diversity of contributors, lower cost, higher performance capability, higher performance reliability and reduced development time lines. This systems has been tested in several thousands of hours of simulation and several hundreds of hours of in-water experiments, on platforms such as the Bluefin 21in. AUV, the Hydroid REMUS-100 and the Remus 600, the Ocean Server Iver2 and the Ocean Explorer 21-in. [14][16] [24].

Teleo-Reactive EXecutive is a software framework (TREX) that creates a single runtime environment for creating 
and executing plans. This means that plans are created, and recreated on a vehicle as it undertakes its mission, enabling replanning to adapt to environmental and vehicle realities. TREX is an instance of a Model-Based architecture, meaning that the knowledge about what is possible is held in a model. In the case of T-REX applied to an AUV this would mean the creation of a model of key parts and relationships for a given vehicle.

MOOS-IvP is a behaviour based architecture. Both made up of middleware, infrastructure toolsets etc. and higher level autonomy capability. Both contain modules that are self contained controllers, MOOS-IvP describes as 'selfcontained mini expert systems' TREX has teleoreactor SPA. Current adaptive AUV control work seems to have come back to this early question of reactive versus deliberative.

\section{FAILURE ANALYSIS}

There are a number of factors that can explain the reason why a new technology is not adopted by a given company or industry. The technology may not be as beneficial as originally thought or it may require unrealistic computer or human overhead. In this section we capture expert belief with regard to which factors may explain the lack of use of mission adaptive planning systems by the wider AUV community. The principle is similar to that followed for accident investigations. Once a timeline event has been established the next phase is to conduct a root-cause analysis. Scenarios representing different hypothesis are captured in an event tree, then experts are interviewed to assess the likelihood of a given scenario or hypothesis leading to the 'accident' [25] - which in this case is failure to adopt mission planning software. Experts' assessments for all hypotheses were aggregated using an unweighted linear pool. However, for this study, we also intend to highlight the difference of opinions across the panel of experts. This is so that requirements critical to each party for technology adoption can be more effectively identified.

In the following subsections we present the event tree derived to capture the main causes for failure to adopt mission planning software. Next the expert judgment elicitation process is described.

\section{A. Event tree of Failure to Adopt Mission Planning systems}

An event tree is a well known technique for modeling probabilistic problems, each event tree represents a unique probabilistic model. The tree is designed from left to right, starting from the End event - which is the failure for which one is looking to find the most likely root causes. The tree for failure to adopt adaptive mission planning systems is presented in Fig. 1. The End event, failure to adopt adaptive mission planning system is the node on the left hand side of the diagram. Chance nodes link the End event to other hypotheses. There is a likelihood associated with each hypothesis, which is obtained from expert assessment - this is discussed in more detail in the following sub-section. The tree was developed following a review of the literature and a discussion with adaptive mission planning researchers and engineers. We have identified the following causes for failure to adopt adaptive mission planning software:
Failure to understand the technology. The engineers working in the AUV industry are trained software or electronic engineers. However the component of training in artificial intelligence is small, sometimes not more than one module in a bachelor's degree. So it is possible that the technology is not adopted because the community of practitioners does not understand the technology.

Failure due to the uncertainty with regards to resulting vehicle performance. The level of uncertainty is a key factor of any decision making process. It is impossible to test all combinations of a software algorithm. For example a small code containing 5 variables, each taking 100 states would require $10^{10}$ runs to test all possible combinations. If each run takes 1 second, the testing time would have to be over 316 years. The computer science community has devised formal methods for software verification and statistical testing techniques to minimize the burden of software testing. Nevertheless this is not sufficient, confidence in new AUV technology is established by the successful number of sea trials. The lack of sea trials may be slowing the wider use of mission adaptive planning systems.

Technology is too expensive. Cost is an important factor in any decision making process. Significant efforts are required to tailor mission adaptive systems to a type of vehicle. Key costs in this system are the development costs, which include computer overhead costs and the uncertainty associated with the development lifecycle. The computer science community is familiar with the Waterflow or $\mathrm{V}$ diagram for system development [26]. The spiral model proposed by Boehm et al. is also known in the computing community, which takes into account the prototyping phase [27]. However the development of knowledge based systems has its own development lifecycle [28]. How this lifecycle can be adopted by the industry may also be an obstacle stopping the adopting mission planning software.

Benefits are not significant. Adaptive mission control has been used for accomplishing very particular tasks, such as to identifying a plume or other feature of interest. There is an argument that some of the rules adopted by the machine can be hardcoded onto the machine and thus similar performance can be achieved without the use of Artificial Intelligence (AI) based techniques. The collision avoidance system for Autosub3 is a clear example where a simple rule based system can perform the task that some would consider being a characteristic of an intelligent system. The Autosub3 collision avoidance navigated its way out of a cavity under an ice shelf in the Pine island glacier. The vehicle managed to navigate its way out after a major incident $55 \mathrm{kms}$ into the ice shelf using non-adaptive rule based techniques [29].

Large uncertainty with regards to legal implications. A potential host of legal concerns related to AUVs irrespective of adaptive mission planning has been documented in [30]. More recently Kirkwood has set out a series of legal questions in response to a number of incidents that occurred with MBARI's Dorado AUV [31]. As vehicles become more autonomous and able to perform longer missions it is possible that vehicles will cross water borders of different countries. The issue of liability 


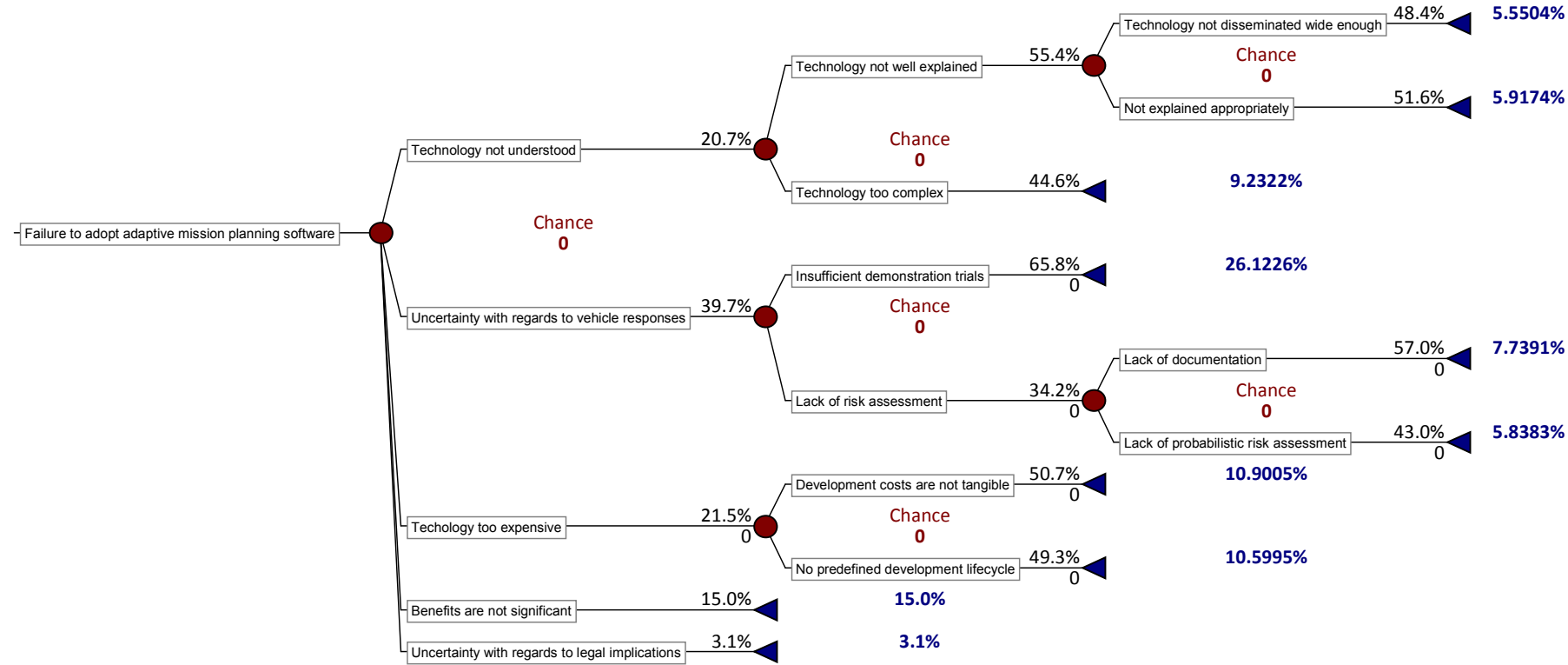

Fig. 1. Event tree of expert judgments into the failure to adopt mission planning systems. Red circles represent chance nodes and blue triangles represent root causes.

concerning a decision taken by an adaptive mission system may also be a deterrent for failure adopt this technology.

Failure modes at level 1 can be decomposed into other subsidiary failure modes at level 2. For example the Technology not understood event can be caused by two events: technology not well explained and technology is too complex.

The first captures issues like how widely the information is disseminated. Many of the publications on the use of adaptive mission planning systems are found in robotics or AI journals and conference proceedings. This literature is not immediately accessible to engineers working in oceanography. Furthermore the language used in the literature may not be easy to understand for people outside the field of artificial intelligence. Thus the hypothesis technology not well understood is further expanded in two events: technology not disseminated wide enough and not explained appropriately.

Other level 1 events that are expanded into level 2 and 3 events are: uncertainty with regards to vehicle responses and technology is too expensive. The description of the level 2 and 3 events follows in the same manner as was described for the level 1 hypothesis.

A key benefit of using the event tree approach is that in addition to identifying the most likely explanation for a failure it helps identify what other events or hypotheses may explain the failure. This is useful information for reducing the risk of future scenarios.

The event tree structure presented in this section was implemented in PrecisionTree5.7, a software tool commercially available to implement decision support models. A discussion of the expert judgment elicitation rationale for the likelihoods of each hypothesis is presented in the following subsection.

\section{B. Expert judgment Elicitation}

After the event tree has been described, the next step in the process is assessing the likelihood for each event. This task was carried out by a group of experts from four countries who have an interest in adaptive systems. Some experts have worked as researchers in the field others are experienced AUV engineers, others are senior managers who have in the past worked as engineers.

Structured expert judgment refers to an attempt to subject the process of eliciting expert judgments to transparent methodological rules, with the goal of treating expert judgments as scientific data in a formal decision making process. The process by which experts come to agree is the scientific method itself. Structured expert judgment cannot preempt this role and therefore it cannot have agreement as its goal [32]. Cooke [32] argues that a structured expert judgment elicitation process may aspire to three different goals: census; political consensus and rational consensus. In this study we aim to meet two of these goals. First we want to conduct a census capturing the general perception with regards to failure to adopt mission planning systems and second we want to present the results of a rational consensus with regards to the most likely root cause for the failure to adopt the technology.

The following experts took part in this judgment elicitation process:

Expert 1 is a professor in marine engineering, works for a government institution, has 14 years experience in AUVs. Expert 1 is based in Australia.

Expert 2 is an experienced robotics engineer and manager with background in AI, works for a private company and has 12 years experience in AUVs. Expert 2 is based in Canada.

Expert 3 is a research engineer with a $\mathrm{PhD}$ from MIT in mission adaptive technology. Expert 3 has 12 years experience with AUVs and has participated in more than $10 \mathrm{AUV}$ 
supported science campaigns. Expert 3 is based in United States.

Expert 4 is a senior research engineer, works for a research laboratory where he is head of a research group responsible for the development of mission adaptive technology tailored to large AUVs. Expert 4 has 7 years experience in AUVs. Expert 4 is based in United States.

Expert 5 works for a research laboratory. Expert 5 has 3.5 years experience in AUVs. Expert 5 has conducted many deployments and is leading the design of a new AUV for a Canadian University.

Expert 6 works for a private company. Expert 6 is mainly a technologist. Expert 6 is a consultant in AUVs; he has worked on high profile AUV deployments, including the deployment of the ISE Explorer in the Arctic. Expert 6 has 6 years experience in AUVs. Expert 6 is based in New Zealand.

Expert 7 works for a research laboratory; previously expert 7 has worked as university lecturer. Expert 7 has 9 years experience with AUVs. Expert 7 is based in Scotland.

Expert 8 works for a research laboratory. Expert 8 is a manager and has 5 years experience with AUVs. Expert 8 is based in United States.

Expert 9 works for a private company. Expert 9 has 10 years experience with AUVs. Expert 9 is based in United States.

Expert 10 is a professor in marine geology, works for an academic institution. Expert 10 has 10 years experience with AUVs. Expert 10 is based in United States.

An online survey was prepared and sent to all experts. The online survey consisted of questions asking the experts to quantify the likelihood of each hypothesis. The survey contained help instructions to clarify the question if required.

Of the 10 experts 5 work for research laboratories, 2 for privately owned companies, 1 for a government institution and 2 work for an university. The combined experience of the experts is 85 years giving an average of experience of 8.5 years; the $75 \%$ quartile is 11.5 years. The role of the experts varied over the years. When asked to provide assessments of their current role one expert stated that he was an engineer, one was manager, one was a technologist. The seven remaining experts stated that they had mixed roles. Fig. 2 presents the assessments for all seven experts concerning their current role. Seven experts have managerial roles. The two experts with significant managerial roles also have a significant amount of their time devoted to engineering tasks (40 and 50\% of their time). This shows that the experts that took part in this study are in a good position to understand the processes needed to adopt new technology.

All experts were asked to quantify the likelihood of each hypothesis. Starting from the point where there is $100 \%$ confidence that failure to adopt adaptive mission system is explained by the five events listed in level 1, each expert was asked to spread the $100 \%$ confidence through the five hypotheses. The same process was followed to assess the likelihood of the events in level 2. Results of these assessments are presented in the next section of the paper.

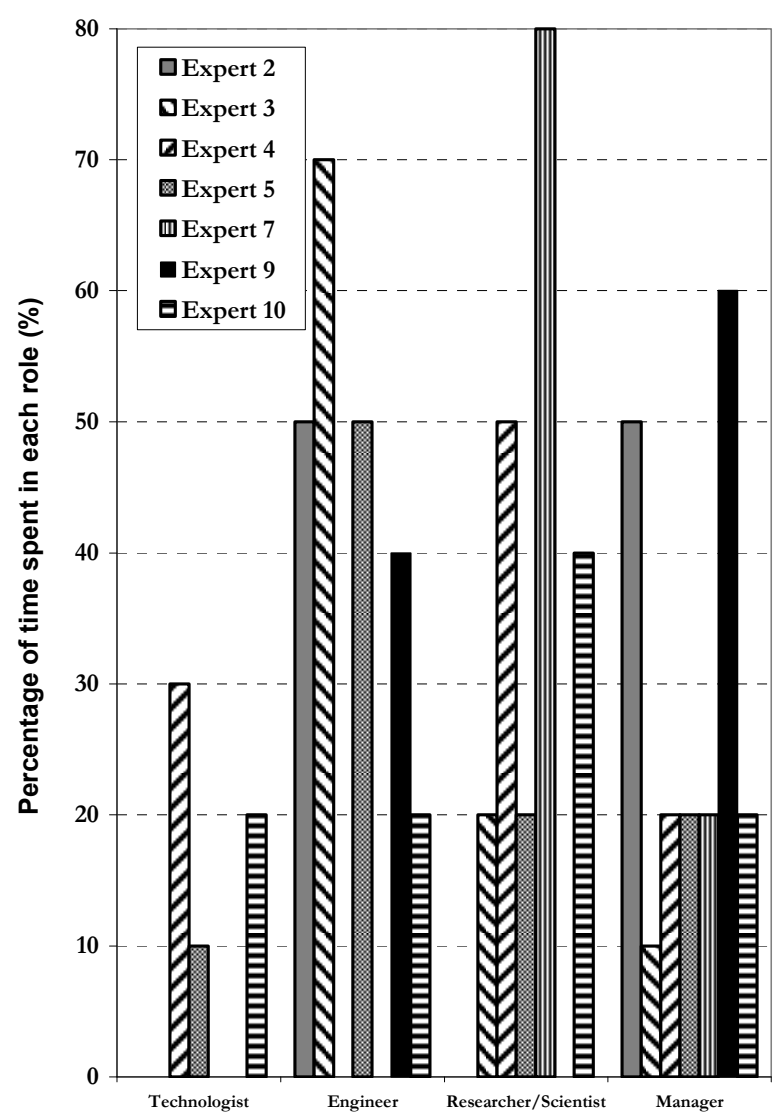

Fig. 2. Experts current roles in AUVs.

\section{ANALYSIS OF THE ASSESSMENTS}

Once all expert assessments have been provided, the next stage is to aggregate the expert judgments in a single assessment that represents the group view. However, as highlighted before the focus of this paper is also to provide a census of the general perception of why there has been a failure to adopt adaptive mission planning software. Therefore in addition to identifying the most likely causes for the failure to adopt adaptive mission planning software we will also discuss the variability in the assessments. The aggregated result of the assessments for each hypothesis is presented in blue in Fig.1.

\section{A. Level 1 failure events}

As shown in Fig.1, at Level 1, the most likely explanation for failure to adopt mission planning software is the uncertainty associated with vehicle behavior. The linear pool gives a $39.7 \%$ confidence that this is the main cause for failure to adopt adaptive mission planning software. Four of the experts (3, 8, 5 and 9) strongly agree with this view by providing their highest assessment to this hypothesis. Expert 3 assigned $75 \%$ to this hypothesis, expert $860 \%$, expert $960 \%$ and expert 5 assigned $40 \%$. Expert 8 briefly summarizes that "... it is not perceived but sometimes actual failure to understand or be able to properly predict and modify behavior for vehicle response". Expert 9 adds that "The asset value is too significant and the requirement for precise position 
prohibits true autonomy. Our use of AUV necessitates great confidence in position."

Expert 7 and 6 have a different view; both argue that costs and lack of clear benefit are the main causes for failure to adopt adaptive mission planning systems. Expert 7 states that within the community of marine scientists there is not sufficient pull to generate the demand for easily integrated solutions, in part because the marine science community would need to define the science goals for the autonomous actions. In his personal experience, steps towards developing autonomy with respect to science goals were relatively expensive with greater technical, rather than scientific, paybacks, thus scientists need to be drawn into this technical development. Expert 6 adds a similar argument, that from a commercial point of view, the value proposition may not be well defined and so the unknowns which have to be solved using manpower and time - have an unknown value, thus cost surpasses value. The lack of understanding of the technology has a direct impact on the perceived costs. These two factors are the main causes for failure to adopt mission adaptive planning systems. Expert 2 argues that the process by which the industry approaches new developments is inhibiting the integration of AI based technology. The general approach is that AUV builders sell existing technology and then wait for customers to ask for more functionality and fund it. This means there is a lack of funds for long term R\&D in autonomy. "The perception is that customers don't need it or want it, but I'm not sure how valid that perception is based on what is conceptually possible."

This view is also supported by expert 4 , who states that "The fundamental issue is that of poor understanding of what "autonomy" (esp. AI based) is, in the first place. Another major issue has to deal with the dearth of technical AI expertise in the ocean sciences/engineering. And funding agencies do not understand the implication of using AI methods towards such stochastic domains where uncertainty can be tackled effectively using such methods."

According to expert 1 failure to understand the technology is also a key factor. Expert 1 assigned 50\% to uncertainty with regards to vehicle response and 50\% to technology not understood. Expert 10 assigned $40 \%$ to both uncertainty with regards to vehicle response and technology too expensive.

The second most likely explanations for failure to adopt mission planning systems are both lack of understanding and technology too expensive. This is general the view of all members of the expert group.

Uncertainty with regards to legal implications is the least likely explanation for failure to adopt mission planning technology. The general view is that legal issues have always been a concern but it has never stopped the use of AUVs.

\section{B. Level 2 failure events}

Some of the events at Level 1 are further decomposed in sub events at level 2. This allows us to have more detail concerning the root cause for failure to adopt adaptive mission planning software. The likelihoods for failure modes at Level 2 are presented in Table 1. At Level 2 'Insufficient demonstration trials' is the main cause for failure to adopt adaptive mission planning software. Five experts strongly agree with this view, experts 2, 3, 6, 7 and 8 assigned likelihoods between $75 \%$ and $90 \%$ to this hypothesis. The idea that seeing is believing is something that resonates with these experts' rationale. Expert 2 commented that "people need to see something in action to really believe in it. Risk analysis is beneficial to ensure a mission will be as successful as possible, but it's not sufficient in its own right." Expert 3 agrees with this view and highlights the difficulty in testing adaptive mission planning systems in real environments: "Testing is difficult and expensive, particularly if interested in a specific phenomenon that is not easily encountered, e.g. hydrothermal vent prospecting. Formal risk assessment is an interesting idea and perhaps applicable in some cases." Table 1 presents the combined assessment for the likelihoods of causes at Level 2.

TABLE I. Root CAUSES AT LEVEL 2

\begin{tabular}{|l|c|}
\hline \multicolumn{1}{|c|}{ Failure mode } & Likelihood \\
\hline Insufficient demonstration trials & $26.1 \%$ \\
\hline Lack of risk assessment & $13.5 \%$ \\
\hline Technology not well explained & $11.5 \%$ \\
\hline Development costs are not tangible & $10.9 \%$ \\
\hline No predefined development lifecycle & $10.6 \%$ \\
\hline Technology too complex & $9.2 \%$ \\
\hline Total & $81.8 \%$ \\
\hline
\end{tabular}

Experts 1 and 10 argue that lack of risk assessment has a strong influence on the perceived uncertainty in the vehicle behavior but still rate the lack of trials as the most influential factor. Both experts assigned $60 \%$ likelihood that the perceived uncertainty with regards to the vehicle response was caused by insufficient demonstration trials. Experts 8 and 9 assigned $50 \%$ to both hypothesis, both rank the lack of demonstration trials as equally influential to the lack of risk assessment. This is a quite interesting assessment, particularly because the experts come from different industrial sectors. Expert 4 has a contrasting view to the rest of the experts prescribing the lack of risk assessment as the main explanation for the perceived uncertainty in vehicle behavior.

As described in the previous section the technology is not well understood because either it is not well explained or because the technology is too complex. The assessments of this statement depend on the type of technique under consideration, as there are some non-complex adaptive mission planning techniques as stated by both experts 3 and 5 . The experts were divided with regards to which of the two factors has more influence on the lack of understanding of the technology. Experts 1, 2, 5 and 9, believe that the technology is not explained appropriately. Expert 2 recognizes that the technology is complex but it could be explained better and thus assigned $60 \%$ to the likelihood of technology not being well explained and $40 \%$ to the likelihood of technology being too complex. Experts 1, 5 and 9 assigned likelihoods between $70 \%$ and $80 \%$ to the technology not well explained hypothesis. Experts 4, 6 and 8 believe that the complexity is the main cause 
for the lack of understanding. Expert 4, a leading scientist in adaptive mission planning, agrees that the level of explanation is often unsatisfactory. According to expert 4 this is caused by the size of the adaptive mission planning system. T-REX is implemented in more than 30,000 lines of code. It is a big challenge to explain the multiple facets, from Planning, to Execution to representation to inference. Experts 7, 3 and 10 assigned $50 \%$ to both hypothesis. Expert 3 has had some success allowing real-time adaptive modification of missions with supervision. So expert 3 does not believe that the complexity is the main issue. Expert 7 is a strong believer that almost anything complex can be explained in understandable terms. Expert 10 agrees that the two hypotheses go hand in hand.

\section{DISCUSSIONS AND CONCLUSIONS}

The lack of sufficient trials is by far the major factor stopping the adoption of adaptive mission planning software. Increasing the number of trial deployments is an obvious recommendation to mitigate this. Making the results of the trial deployments public and widely accessible is of equally great importance. One key point here is what makes a trial a successful test? Is it the identification of a given target or feature? is it the time taken to find the target? the number of attempts? These criteria need to be defined before the AUV community starts discussing validation of the technology. Once criteria have been defined then it becomes a question of identifying the number of successful tests to statistically quantify the confidence that given criteria is met. To address this, one or more groups with well established capability for adaptive mission planning could offer to run missions with objectives and criteria set by groups not having that capability, as wider demonstrators. Such a set of actions will increase the community confidence in the precision of adaptive mission planning systems.

Adaptive mission sampling strategies are often highly tuned algorithms, to quote expert 10 "much like a tightly tuned PID controller and therefore work very well in the conditions and vehicles/settings for which they are initially developed but struggle in new settings- say differences in bottom type, morphology, vehicle make etc." In our review we have identified that some frameworks require training for the knowledge based system to be effective. The process for doing the training needs to be transparent as does the method to measure its performance.

Formal risk assessment has been identified as a key factor regardless of the level of autonomy [33][34][35]. When considering the adaptive nature of the mission then estimating the reliability of the system is key for the AUV risk assessment process. Well accepted methods for devising software tests for safety critical systems can be applied to estimate the reliability of adaptive mission systems [36].

Identifying who is at fault for the lack of use of adaptive mission systems, whether it is the industry that has a lack of interest in investing in a technology that does not bring short term benefit or whether it is the researchers that have not explained the methods appropriately is not the aim of this paper. However, as identified by the panel, there is a general perception that the costs are too high and this may be due to a lack of understanding. A method to counter this is the combination of an increased effort to disseminate the results in oceanography journals and conferences, the frequent presentation of tutorials in conferences would be of great help.

It is worth noting that adaptive mission planning has advanced much further in the unmanned air vehicle (UAV) and unmanned ground vehicle (UGV) environments than in UAV. This is largely because the vehicles are less expensive to build, there are no pressure hulls, exotic batteries and expensive sensors; and in addition the vehicles are much easier to find if they get lost. For an AUV, an Iridium modem just signals the vehicle position and other relevant info to the supervisor who may be thousands of miles away. The fact that physics prevents us from enjoying these same capabilities in AUVs explains why managers are unwilling to risk their multi million dollars machines for the sake of scientific research, particularly when they can accomplish their immediate objectives without doing so.

Having recognized this problem now we in the AUV community need to fix it. Until we start doing something, we will not be on the path that leads to long term autonomy and the ability to implement increasingly complex missions in unstructured environments. The benefits of being able to do this are indeed very significant. For example, along with more efficient power sources, this capability is what will ultimately enable Long Duration AUVs and the truly autonomous work vehicle. So the industry needs to be paying a lot more attention to what university labs the world over are doing with toy-size robots, and look at how they can recreate the results in swimming pools, then in open-water under controlled conditions.

Adaptive planning and control systems are not yet sufficiently mature for use in operational AUVs. Because of the substantial benefits that this technology can provide to subsea vehicle missions, AUV researchers, operators and managers need to place a higher priority on its development and introduction into open-water operations.

\section{ACKNOWLEDGMENT}

The ECOR panel would like to acknowledge Kanna Rajan for providing insights concerning the development and use of T-REX. The panel would also like to thank all 10 experts that took time to fill in the online survey. Their pro-active engagement in this survey was a major contribution for the research presented in this publication.

\section{REFERENCES}

[1] U.S. Commission on Ocean Policy, "An Ocean Blueprint for the 21st Century," Final Report of the U.S. Commission on Ocean Policy,2005.[Online]

http://www.oceancommission.gov/documents/full color rpt/27 chapter27.pdf Accessed on 20th August 2012.

[2] Vincent Rigaud, JL Michel, James Ferguson, JM Laframboise, T. Crees, Pierre Leon, J. Opderbecke, and Yves Chardard. First Steps in Ifremers Autonomous Underwater Vehicle Program-A $3000 \mathrm{~m}$ Depth Operational Survey AUV for Environmental 
Monitoring. In Proc. 14th Int. Offshore and Polar Eng. Conf., ISOPE, vol. 2, pp 203-208, 2004.

[3] Curtin T.B., Crimmins, D.M., J A Curcio, Michael R Benjamin, and C. Roper. Autonomous underwater vehicles: trends and transformations. Mar Technol Soc J, vol. 39, no. 3:65-75, 2005.

[4] P. Ramos, S.R. Cunha, M.V. Neves, F.L. Pereira, I. Quintaneiro, "Sewage outfall plume dispersion observations with an autonomous underwater vehicle," Water Sci Tchnol, vol. 52, no. 12, pp. 283-90. 2005

[5] Z. Yanwu, R.S. McEwen, J.P. Ryan, J.G. Bellingham, "An adaptive triggering method for capturing peak samples in a thin phytoplankton layer by an autonomous underwater vehicle," OCEANS 2009, MTS/IEEE Biloxi - Marine Technology for Our Future: Global and Local Challenges , pp.1-5, 26-29 Oct. 2009

[6] P. Patrón, E. Miguelañez, Y. Petillot, D.M. Lane and J. Salvi, “ Adaptive mission plan diagnosis and repair for fault recovery in autonomous underwater vehicles";IEEE Oceans 2008

[7] A.M. Meystel, and J.S. Albus, "Intelligent systems: architecture , design, and control," Wiley Interscience, 2002,

[8] J. S. Albus, R. Quintero, and R. Lumia. An Overview of NASREM: The NASA/NBS Standard Reference Model for Telerobot Control System Architecture, NASA STI/Recon Technical Report N, 95:12854, 1994.

[9] H. Hillaker. Tribute To John R. Boyd. Code One Magazine, July 1997.

[10] J.R. Boyd. The essence of winning and losing. Lecture notes, Unpublished, 1996.

[11] P. Patron, E. Miguelanez, Y.R. Petillot and D.M. Lane, "Fault tolerant adaptive mission planning with semantic knowledge representation for autonomous underwater vehicles," In Proc. IEEE/RSJ Int. Conf on Intelligent Robots and Systems, pp.25932598, 22-26 Sept. 2008

[12] P. Patron and D.M. Lane. Adaptive mission planning: the embedded OODA loop. In Proc 3rd SEAS DTC Technical Conference, 2008.

[13] P. Patron, E. Miguelanez, Y.R. Petillot, D.M. Lane and J. Salvi, "Adaptive mission plan diagnosis and repair for fault recovery in autonomous underwater vehicles," In Proc. IEEE Oceans Quebec, 2008.

[14] J.M. Evans, P. Patron, B. Smith and D.M. Lane, "Design and evaluation of a reactive and deliberative collision avoidance and escape architecture for autonomous robots," Auton Robot, vol. 24, no. 3, 2008, pp. 247-266.

[15] F. Dabe, M. Barbier, H. Ayreault, S. Nicolas and G. Kermet, "Goal driven planning and adaptivity for AUVs," cert.fr, vol. 5, pp.21-24, August 2005.

[16] M.R. Benjamin, H. Schmidt, P.M. Newman, J.J. Leonard, "Nested autonomy for unmanned marine vehicles with MOOSIvP," J Field Robot, vol. 27, no. 6, 834-875, 2010.

[17] C. McGann, F. Py, K. Rajan, H. Thomas, R. Henthorn, and R. McEwen "T-REX: A deliberative system for AUV control. ICAPS WS on Planning and Plan Execution for Real-World Systems," Providence, RI, USA, 2007.

[18] R.M. Turner, "Context-sensitive, adaptive reasoning for intelligent AUV control: Orca project update," In Int. Symp. on Unmanned Untethered Submersible Technol., pp.426-435, 1995.

[19] K. DeMarco, M.E. West, T.R. Collins, "An implementation of ROS on the Yellowfin autonomous underwater vehicle (AUV)," OCEANS 2011, pp. 1-7, 19-22 Sept. 2011.
[20] S.M. Veres, L. Molnar, N. Lincoln and C. Morice, "Autonomous vehicle control systems - a review of decision making," P I Mech Eng I-J Sys, vol. 225, no. 2, 155-195. 2011.

[21] A. Jonsson, P. Morris, N. Muscettola, K. Rajan and B. Smith, "Planning in Interplanetary Space: Theory and Practice," Proc. Fifth International Conference on Artificial Intelligence Planning Systems, Breckenridge, CO, USA, April 14-17, 2000.

[22] J. Frank and A. Jonsson, "Constraint-based Attribute and Interval Planning,” Constraints, vol. 8, no. 4, pp. 339-364, 2003.

[23] K. Rajan, F. Py, C. McGann, J. Ryan, T. O'Reilly, T. Maughan and B. Roman, "Onboard Adaptive Control of AUVs using Automated Planning and Execution," Int. Symp. on Unmanned Untethered Submersible Technology (UUST), Durham, NH, pp. 13, August 2009.

[24] D. Wang, P.F.J. Lermusiaux, P.J. Haley, D.L. Eickstedt, G. Wayne, H. Schmidt, "Acoustically focused adaptive sampling and on-board routing for marine rapid environmental assessment,” J Marine Syst, vol. 78, pp. S393-S407, 2009.

[25] J.E. Strutt, "Report of the inquiry into the loss of Autosub2 under the Fimbulisen." National Oceanography Centre Southampton Research and Consultancy Report, 12, 39pp 2006.

[26] R. Winston "Managing the Development of Large Software Systems", In Proc. of IEEE WESCON 26 (August).pp. 1-9, (1970),

[27] B.W. Boehm,, "A spiral model of software development and enhancement," Computer, vol.21, no.5, pp.61-72, May 1988.

[28] K.B. Korb and A.E. Nicholson "Bayesian Artificial Intelligence. Computer Science and Data Analysis." CRC/Chapman Hall, Boca Raton, 2004.

[29] M. P. Brito and G. Griffiths, "Results of expert judgments on the faults and risks with Autosub3 and an analysis of its campaign to Pine Island Bay, Antarctica, 2009," In, Proc. of the International Symposium on Unmanned Untethered Submersible Technology (UUST 2009), Durham, New Hampshire, 23-26 August 2009. UUST 2009 Durham NH, USA, Autonomous Undersea Systems Institute (AUSI), [14p]. 2009

[30] Society of Underwater Technology, "The Operation of Autonomous Underwater Vehicles, Volume One: Recommended Code of Practice for the Operation of Autonomous Marine Vehicles," Second Edition

[31] W. Kirkwood, "AUV Incidents and Outcomes" IEEE - MTS Oceans, Biloxi, Mississippi, Oct. 28, 2009.

[32] R.M., Cooke, L.H.J. Goossens, "TU Delft Expert Judgment Data Base," Special issue on expert judgment Reliab Eng Syst Safe, vol. 93, pp. 657-674, 2008.

[33] M.P. Brito, G. Griffiths and P. Challenor, "Risk Analysis for Autonomous Underwater Vehicle Operations in Extreme Environments," Risk Anal, vol. 30, no. 12, 1771-1788. 2010.

[34] M. Brito and G. Griffiths, "A Markov Chain state transition approach to establishing critical phases for AUV reliability," IEEE J Oceanic Eng, vol. 36, no. 1, 139-149.

[35] M. Brito, G. Griffiths, J. Ferguson, D. Hopkins, R. Mills, R. Pedersen and E. MacNeil, "A Behavioral Probabilistic Risk Assessment Framework for Managing Autonomous Underwater Vehicle Deployments," J Atmos Ocean Tech, in press.

[36] JA Whittaker, M.G. Thomason, "A Markov chain model for statistical software testing," IEEE T Software Eng, vol. 20, no. 10, pp. 812 - 824, October 1994. 